\title{
http://bjas.journals.ekb.eg \\ Combination of lactic acid 15\% and Trichloro-Acetic Acid 3.75\% \\ Chemical Peeling Versus Platelet-Rich Plasma in Treatment of Infraorbital Dark Circles
}

H.H.Sabry, A.I.Mustafa, S. H.Eldein

Dermatology, Venereology and Andrology Dept., Faculty of Medicine, Benha Univ., Benha, Egypt

E-Mail:Samar6@gmail.com

\begin{abstract}
While compound stripping has been utilized to treat an assortment of facial pigmentary messes, there are no randomized-control concentrates on the adequacy of substance stripping for the treatment of periocular hyperpigmentation. We intended to assess the adequacy and wellbeing of shallow compound stripping procedure comprising of blend of Lactic corrosive (LA) $15 \%$ and Trichloroacetic corrosive (TCA) $3.75 \%$ in gel recipe and contrasting it and intradermal infusion of PRP in the treatment of inrfaorbital dark circles. 60 patients experiencing periorbital dark circles (PODC)was devided to 2 geoups of treatment. Gathering A: Thirty patients with periorbital dark circles and skin types III, or IV were remembered for the investigation. Patients will be treated with effective use of Lactic corrosive $15 \%$ and TCA $3.75 \%$ in gel equation. The ffect was photograph reported, and a patient's and doctor's worldwide appraisal was assessed. Gathering B: Another 30 will be treated with intradermal autologous platelet rich plasma infusion into the periorbital zone. The treatment course will comprise of six meetings once every other week. the level of progress of PODC rewarded with concoction stripping in bunch A was seen as incredible in $16.70 \%$ of the patients, while indicating great in half, reasonable and helpless outcome in $20 \%$ and 13.30 of them, separately. None of the patients' skin turned out to be more regrettable or didn't react to the treatment. Measurably huge improvement in the evaluation of PODC was accounted for $(p<0.004)$. We presumed that the two strategies for treatment were similarly viable in the treatment of PODC, with the improvement of PODC saw from the main treatment meeting for both substance stripping and carboxytherapy. Compound stripping was greatly improved in the treatment of pigmented sort of PODC, with insignificant endured reactions.
\end{abstract}

\section{Introduction}

When all is said in done, treatment with depigmenting operators must be proceeded for a while before restorative advantages are acquired. The activity components of depigmenting specialists are restraint of tyrosinase action, hindrance of DNA union in hyperactive melanocytes, decrease of epidermal substance of melanin, and thickening of the epidermis (granular layer) [1].

While substance stripping has been utilized to treat an assortment of facial pigmentary messes, there are no randomized-control concentrates on the adequacy of concoction stripping for the treatment of periocular hyperpigmentation. The stripping operators expel melanin from the layer corneum and epidermis. Profound strips may expel melanin from dermis however may prompt dyspigmentation and scarring. Here the skin is flimsy, so profound strips are not suggested [2]. Lasers have progressively been utilized in corrective dermatology. Dark circles because of unreasonable pigmentation have been effectively rewarded with different shade lasers [3].

Treatment of infraorbital dark circles identified with tear trough is increasingly intricate. It might require intrusive surgeries to lift the delicate tissues from the fundamental maxilla, fat transplantation or fat expulsion, and septal resection [4].

Platelet-Rich Plasma (PRP)' is a general term used to portray hanging plasma that has been acquired from entire blood with grouping of platelets higher than typical focus found in the flowing blood. PRP's work instrument relies upon the way that platelets contain significant substances called (development factors), which have a known job during the time spent change and recharging of tissues [5].

The point of the current examination was to assess the adequacy and security of shallow substance stripping strategy comprising of blend of Lactic corrosive (LA) $15 \%$ and Trichloroacetic corrosive (TCA) $3.75 \%$ in gel recipe and contrasting it and intradermal infusion of PRP in the treatment of inrfaorbital dark circles.

\section{Patient and method}

This examination was done on 60 patients experiencing periorbital dark circles (PODC), enrolled from the Out Patient Clinic of Dermatology, Venereology and Andrology Department of Benha University Hospital from January 2019 to August 2019. All patients got total data on steps of treatment and numerous treatment meetings were important for fulfillment. 
Consideration standards was mellow to serious infraorbital dark circles and Photo skin type III and IV. While Exclusion criteria was history of keloidal propensity history of draining or platelet issue, history of significant medical procedure in recent months, Presence of any intense disease on face like herpes and folliculitis, Patients with HIV, HBsAg, or any incessant sickness, Pregnancy.

All patients will be exposed to finish history taking, total general assessments , Dermatological assessments were finished including Skin phototyping as indicated by Fitzpatrick's skin type order [6] and manual extending of the lower eyelid skin can assist with separating between obvious pigmentation and shadowing impact.

Tolerant are partitioned to 2 gatherings Group An A mix of stripping specialists comprising of TCA in the convergence of $3.75 \%$ and LA of $15 \%$ was set up in a gel recipe.

Gathering B rewarded by infusion of PRP on the periorbital region. Six meetings were intended to be performed on each side with 2week spans in PRP.
After treatment patients are told to photograph security was by sunscreen with SPF 50 , skin anti-infection cream and shirking of bothering cleansers. All patient were protested ( Pain appraisal, Patient fulfillment, Evaluation of treatment and Side impacts)

The gathered information was amended, coded, classified and acquainted with a PC utilizing Statistical bundle for Social Science (IBM Corp. Discharged 2017. IBM SPSS Statistics for Windows, Version 25. Armonk, NY: IBM Corp.).

\section{Results}

Grades of dark circles were assessed, $25 \%$ had grade I, $46.7 \%$ had grade II, $28.3 \%$ had grade III.

Onset was gradual in all cases. Course was stationary in $31.7 \%$ and progressive in $68.3 \%$. Mean disease duration was 6.4 years.

No significant differences were found regarding a dark circles' features between studied groups.

Better response was significantly associated with $\mathrm{CP}$ group when compared to PRP group $(\mathrm{p}=0.038)$.

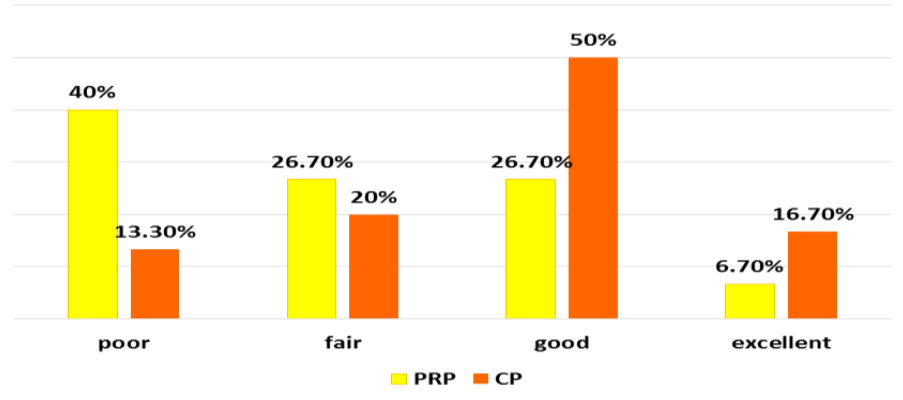

Fig (1) Response among studied groups.

Frequency of pain sensation was associated with PRP when compared to CP group ( $\mathrm{p}=0.007)$. In addition, moderate pain was associated with PRP when compared to CP group ( $\mathrm{p}=0.034)$.

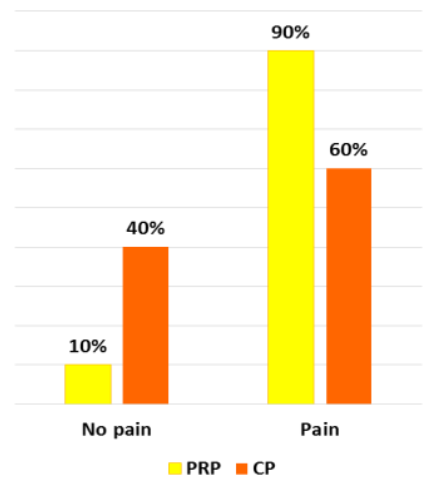

Fig (2) Pain assessment among studied groups.

Better satisfaction was significantly associated with CP group when compared to PRP group $(\mathrm{p}=0.003)$. 


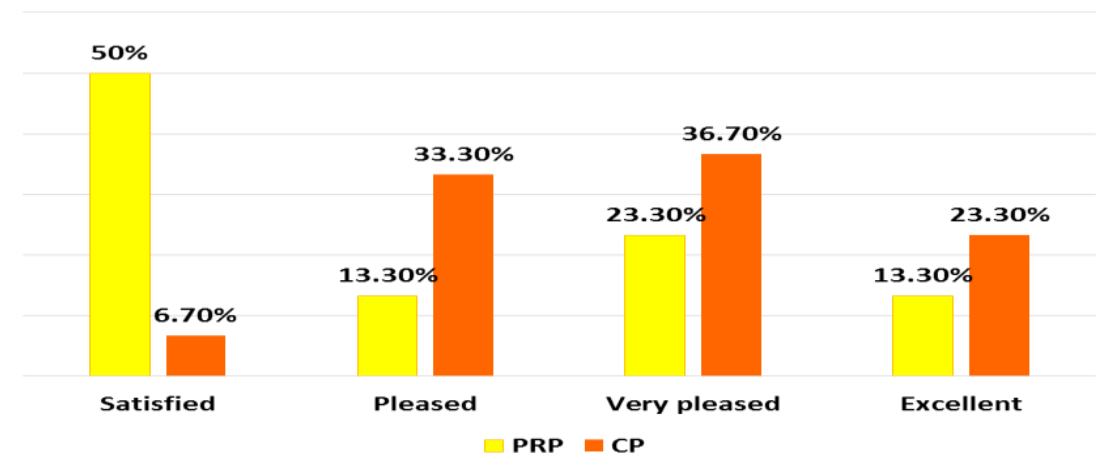

Fig (3) Patients' satisfaction in all studied groups.

Patients' satisfaction showed significant positive correlation with improvement in PRP and CP groups. Otherwise, no significant correlations were found between improvement versus age, grade, course, duration.

Table (1) Correlation of improvement with other studied parameters in studied groups.

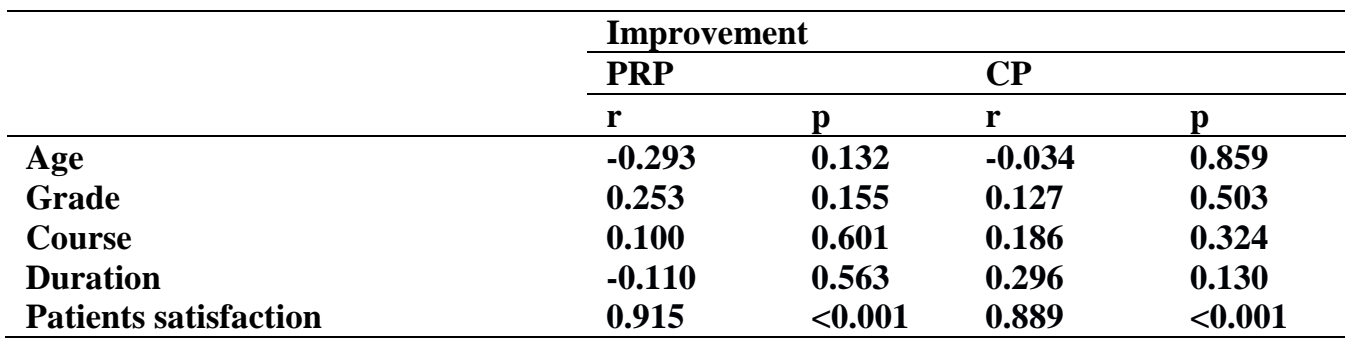

\section{Discussion}

In the current examination, the level of progress of PODC rewarded with compound stripping in bunch $\mathrm{A}$ was seen as superb in $16.70 \%$ of the patients, while demonstrating great in half, reasonable and helpless outcome in $20 \%$ and 13.30 of them, separately. None of the patients' skin turned out to be more regrettable or didn't react to the treatment. Factually huge improvement in the evaluation of PODC was accounted for $(p<0.004)$. This outcome was far and away superior to the consequences of a past report by Vavouli [7]. who performed synthetic stripping with a similar compound mix for infraorbital dark circles. They detailed an astounding reaction in $16.6 \%$ of the patients, while $40.0 \%, 36.7 \%$ and $6.6 \%$ of them indicating great, reasonable and helpless outcome individually.

In the current examination, $23.30 \%$ of the considered patients had great fulfillment with the outcomes, $36.70 \%$ were very pleassed, $33.30 \%$ were satisfied and $6.70 \%$ were fulfilled. This was in concurrence with the investigation by Vavouli [7] in which they revealed that the patient fulfillment result was $63.3 \%$ reacted to be exceptionally fulfilled, with somewhat fulfilled in $23.3 \%$ of them, while the other $13.3 \%$ were disappointed.
In this investigation, the patient worldwide resilience was seen as $40 \%$ encountered no agony felt, $60 \%$ detailed nearness of torment , $20 \%$ felt gentle torment and $3.30 \%$ moderate torment in the patients. This finding was as per Vavouli [7] who revealed $46.7 \%$ astounding resilience in the patients, while $30.0 \%$ and $23.3 \%$ of them indicated great and reasonable resistance, individually.

The symptoms were negligible and recorded as transient in the patients. $46.6 \%$ of patients experienced consuming sensation, $40 \%$ had shivering, $33.3 \%$ gave ery-thema, $20 \%$ showed tingling and the other $20 \%$ had skin dryness. Simultaneously, Vavouli [7] found a similar symptoms which were not considered as re-strictive factor to the patients. This was the primary examination to discover that this mix of synthetic stripping was altogether substantially more compelling for PODC of pigmented type when contrasted with different kinds. This could be clarified by the way that TCA application on the skin produces injury to the epidermis and upper dermis, just as coagulation of skin proteins and melanin scattering.

This is trailed by recovery of new cells with expanded collagen arrangement, which prompts the expansion in the volume of epidermis and dermis. Numerous components 
influence the profundity of skin putrefaction, for example, the expansion in the convergence of TCA, utilization of TCA on a progressively porous skin, increment in the quantity of skin layers utilized in a similar treatment meeting, prolongation of contact time with the skin, the quantity of recently performed meetings, the length among meetings, and the patient's skin type [8].

Lactic corrosive is an alpha hydroxy corrosive (AHA) which encourages desquamation of epidermal cells, melanin scattering, and prompts collagen and glycosaminoglycan affidavit.

In addition, it has been accounted for to hinder tyrosinase compound; along these lines it was utilized in the treatment of melasma [9].

It instigates the skin-helping impact through three components, specifically the enlistment of keratinocytes desquamation and the evacuation of melanosomes; the hindrance of tyrosinase; and the thickening of the epidermis and the dermis, so that the vasculatures become less noticeable [10].

There are a few favorable circumstances of the blend of synthetic strips for example, expanding the profundity of skin infiltration without expanding the centralization of concoction strips; bringing down the treatment's unfavorable impacts, for example, scarring and lasting depigmentation by utilizing low grouping of the stripping operators upgrading tissue recovery and lessening ensuing recuperation time [7].

Also, this compound strip is dependable and a successful strategy for treatment for PODC, particularly the pigmented type. It is less expensive when contrasted with different strategies. It has the upside of wellhomogenizing impact. It is likewise compelling, safe, and very much endured, which is fulfilling for the two patients and doctors [7].

Be that as it may, the main restriction for this sort of treatment is that the patients ought to maintain a strategic distance from sun introduction.

The level of progress of PODC for bunch B rewarded by platelet-rich plasma was brilliant in $6.70 \%$ of the patients, great in $26.70 \%$ while demonstrating reasonable and helpless outcomes in $26.70 \%$ and $40 \%$ of them, separately. None of the patients' skin was declining or not reacting to the treatment. A measurably critical improvement in the PODC grade was accounted for $(\mathrm{p}<0.001)$. The patient fulfillment result was as per the following: $13.30 \%$ announced superb , $23.30 \%$ satisfied, $13.30 \%$ satisfied and half fulfilled. Then again, the patient worldwide resistance was as per the following: $10 \%$ no torment, $90 \%$ announced an agony, $60 \%$ gentle torment and $30 \%$ moderate torment. The reactions were insignificant and recorded as transient, with $33.3 \%$ of the patients having torment at the site of infusion, $26.6 \%$ having edema, $20 \%$ experiencing consuming sensation and $6.6 \%$ showing ecchy-mosis, while two patients didn't report any grumblings.

Al-Shami [11] contemplated the viability of platelet-rich plasma in the treatment of periorbital hyperpigmentation and announced as follows: two patients $(4 \%)$ revealed magnificent improvement, six patients $(12 \%)$ huge improvement, twenty three patients (46\%) moderate improvement, and nineteen patients $(38 \%)$ gentle improvement in the presence of the dark circles.

In that review, the patients were solicited to assess their own level from fulfillment by giving themselves a score from 0-4 focuses and the score was converted into a range from no change to fantastic. For a situation report, melasma was additionally answered to be effectively rewarded with PRP with a perception of $>80 \%$ decrease in epidermal hyperpigmentation [12].

In any case, it was not revealed what the scale they utilized for the perception [12].

Likewise, the strategy of assessing tolerant fulfillment in these current investigations remains very weak. We utilized FACE-Q in our patients when application for the appraisal. The FACE-Q is a patient-revealed result instrument that gauges the experience and results of stylish facial techniques from the patient's point of view giving remarkable knowledge into their fulfillment and wellbeing related personal satisfaction [13].

In methodology focusing on stylish improvement, persistent impression of the treatment result seems, by all accounts, to be most significant in light of the fact that it directly affects patients' self-perception and confidence [14].

A few parts of our investigation might be viewed as amazing. The assessment of fulfillment with facial appearance and skin when the methodology makes it more clear how much the dull eye circles influence the facial appearance and skin fulfillment in patients. While the social and mental capacity evaluations were analyzed, it was seen that the personal satisfaction of the patients had changed after the treatment with PRP for dull eye circles.

It tends to be included that dull eye hover treatment with PRP even adds to the expanded nature of patient-saw age feeling. At the point when the fulfillment with result is analyzed, 
the fulfillment of the patients from the PRP methodology for the treatment of the dull eye circles was seen to be a score of 83 inside the scope of 0-100 and could be deciphered as a decent fulfillment level.

This was the primary investigation to analyze between concoction stripping and PRP in the treatment of PODC. Substance stripping and PRP are helpful medicines of dark circles.

\section{References}

[1] F.Prignano, J.P.Ortonne, G.Buggiani. Therapeutically approaches in melasma. DermatolClin, Vol. 25, PP.337-42,2007.

[2] B.Taskin. Periocular Pigmentation: Overcoming the Difficulties. Pigmentary Disorders, Vol. 2, PP.159,2015.

[3] W.Manuskiatti, R.E.Fitzpatrick , M.P.Goldman. Treatment of facial skin using combinations of $\mathrm{CO} 2$, Qswitchedalexandrite, flash lamp-pumped pulsed dye, And Er: YAG lasers in the same treatment session. J Dermatol Surg, Vol. 26,114-120,2000.

[4] F.E.J.r.Barton, R.Ha , M.Awada. Fat extrusion and septal reset in patients with the tear trough triad: acritical appraisal. Plast Reconstr Surg, Vol. 113, PP.21152121,2004.

[5] J.1.Alsousou, A.Ali, K.Willett , P,Harrison. The role of platelet-rich plasma in tissue regeneration.Platelets, Vol. 24(3), PP.173-82,2013.

[6] T.B.Fitzpatrick. The validity and practicality of sun-reactive skin types i through vi. Archives of Dermatology 124, Vol. (6), PP. 869-871,1988.

[7] C.Vavouli, A.Katsambas, S.Gregoriou, A.Teodor, C.Salavastru, A.Alexandru, G.Kontochristopoulos. Chemical peeling with trichloroacetic acid and lactic acid for infraorbital dark circles.Journal of cosmetic dermatology, Vol. 12, PP. 204209,2013.

[8] E.Y.Chun, J.B.Lee, K.H. Lee. Focal trichloroacetic acid peel method for benign pigmented lesions in darkskinned patients. Dermatol Surg, Vol. 30(4), PP.512-516,2004.

[9] A.Usuki, A.Ohashi, H.Sato, Y.Ochiai, M.Ichihashi. The inhibitory effect of glycolic acid and lactic acid on melanin synthesis in melanoma cells. Exp Dermatol, Vol.12(Suppl 2), PP.43$50,2003$.

[10] K.E.Sharquie, M.M.Al-Tikreety, S.A.AlMashhadani. Lac-tic acid chemical peels as a new therapeutic modality in melasma in comparison to Jessner's solution chemical peels. Dermatol Sur, Vol. 32(12), PP.1429-1436,2006.

[11] S.H.Al-Shami. Treatment of periorbital hyperpigmentation using platelet-rich plasma injections. Am J Dermatol Venereol, Vol.3(5), PP.87-94,2014.

[12]M.Cayırlı, E.Calışkan, G.Açıkgöz, A.H.Erbil, G.Ertürk. Regression of melasma with platelet-rich plasma treatment. Ann Dermatol, Vol.26 (3), PP.401-2,2014.

[13] Memorial Sloan-Kettering Cancer Center:FACE-Q. A revolutionary patient-reported outcome instrument, 2019.

[14] S.J.Al-Saedy, M.M.Al-Hilo, S.H.AlShami. Treatment of acne scars using fractional Erbium: YAG laser. Am J Dermatol Venereol, Vol.3(2), PP.439,2014 